\title{
Discrete Fučík spectrum - anchoring rather than pasting
}

\author{
Petr Stehlík
}

In 1979, deep in the dark days of the Soviet occupation of Central and Eastern Europe, a car with a Belgian couple is crossing the heavily guarded border between West Germany and Czechoslovakia. In their luggage they hide a package with a big amount of cash. If revealed, Jean Mahwin and his wife would end up in a serious trouble. Custom officers wouldn't believe the true story about a donation collection of West European mathematicians for the widow of the recently deceased young mathematician Svatopluk Fučík. The organizer of the collection is none other than Jean Mawhin himself. The driver not only brings dollars, but also brightens faces of hundreds of decent people who learn about this story. Even today. Mathematicians are commonly depicted as out-of-touch and asocial beings. Few people would connect them to acts of courage and compassion. Jean Mawhin defies this stereotype more than anyone else. I am very happy that I can offer my wishes to his 70th birthday. Happy birthday!

"Correspondence: pstehlik@kma.zcu.cz Department of Mathematics, University of West Bohemia, Univerzitni 22, Pilsen, 30614, Czech Republic

\begin{abstract}
In this short note we study a simple discrete Fučík spectrum. Trying to imitate standard continuous pasting procedures, we derive a more complicated discrete analogue - anchoring. Using this technique, we show that the problem of finding the parametrization of the second discrete Fučík branch is equivalent to solving a transcendent equation $A \sin (x)=\sin (B x)$. Based on this equivalence, we state a conjecture that already the second branch has no elementary parametrization, i.e., it cannot be described by a finite number of elementary functions.
\end{abstract}

MSC: $39 \mathrm{~A} 12 ; 34 \mathrm{~B} 15$

Keywords: Fučík spectrum; nonlinear difference equation; resonance; boundary value problem; elementary functions; second branch

\section{Introduction}

Comparison of related continuous and discrete nonlinear problems reveals a very interesting relationship between these two worlds. In some cases, the finite dimension of discrete function spaces could significantly simplify analysis and provide general results (see [1-3]). In other situations, the broken discrete topology, in which sequences or vectors appear instead of continuous curves, causes difficulties without analogies in the continuous world. The goal of this paper is to show that the Fućík spectrum is one of the most astonishing examples of the latter type.

The nonlinear generalization of the eigenvalue problem for ODEs by Svatopluk Fučík [4] was quickly applied in the theory of semilinear boundary problems (e.g., [5, 6]). Since then this concept has been extended to more complicated differential operators (e.g., [7, 8]) and studied in general settings of Banach spaces (e.g., [9]). Attempts to analyze the Fučík spectrum for matrices and difference operators have been less successful (see [10-

\section{焦 Springer}

(c) 2013 Stehlík; licensee Springer. This is an Open Access article distributed under the terms of the Creative Commons Attribution License (http://creativecommons.org/licenses/by/2.0), which permits unrestricted use, distribution, and reproduction in any medium, provided the original work is properly cited. 
13]). Those works reveal the complexity of the matrix problem, which prevents to fully describe the spectrum beyond $2 \times 2$ matrices.

One possible answer to these complications could be to concentrate on a special class of matrices corresponding to specific difference operators. In this brief note, we follow this avenue and try to apply Fučík's pasting technique [4] to the simplest discrete problem, a direct counterpart of the original continuous problem

$$
\left\{\begin{array}{l}
-\Delta^{2} x(k-1)=\mu x^{+}(k)-v x^{-}(k), \quad k=1,2, \ldots, N, \\
x(0)=0=x(N+1) .
\end{array}\right.
$$

Assuming that $x^{ \pm}(k):=\max \{ \pm x(k), 0\}$, we seek the Fučík spectrum, i.e., pairs $(\mu, v) \in \mathbb{R}^{2}$ such that the problem (1) has a nontrivial solution.

In Section 2, there is a short summary of continuous pasting technique. In Section 3, we deal with the trivial first branch of (1). In Section 4, we show that (i) one should rather talk about anchoring than pasting in the case of the second branch, and that (ii) the problem of finding its parametrization is equivalent to the problem of solving $A \sin (x)=\sin (B x)$ with $A<0$ and $B \in \mathbb{Q}$. Finally, in Section 5 we state and discuss the conjecture that the parametrization of the second-branch of (1) is not elementary, i.e., it cannot be described by a finite number of elementary functions.

\section{Pasting in continuous case}

First, let us briefly recall the pasting technique for the original continuous problem [4]

$$
\left\{\begin{array}{l}
-x^{\prime \prime}(t)=\mu x^{+}(t)-v x^{-}(t), \quad t \in(0, \pi), \\
x(0)=0=x(\pi) .
\end{array}\right.
$$

Lemma 1 The piecewise nonlinear BVP (2) has a nontrivial solution if and only if $(\mu, \nu) \in$ $\Sigma^{c}$, where $\Sigma^{c}=\bigcup_{n=1}^{\infty} C_{n}^{c}$, where

$$
\begin{aligned}
& C_{1}^{c}=\{(\mu, v):(\mu-1)(v-1)=0\}, \\
& C_{2 k}^{c}=\left\{(\mu, v): \frac{1}{\sqrt{\mu}}+\frac{1}{\sqrt{v}}=\frac{1}{k}\right\}, \\
& C_{2 k+1}^{c}=\left\{(\mu, v): \frac{1}{\sqrt{\mu}}+\frac{1}{\sqrt{v}}=\frac{1}{k}-\frac{1}{k \sqrt{\mu}} \text { or } \frac{1}{\sqrt{\mu}}+\frac{1}{\sqrt{v}}=\frac{1}{k}-\frac{1}{k \sqrt{v}}\right\}
\end{aligned}
$$

for $k=1,2, \ldots$

Proof The detailed proof could be found, e.g., in [6, Chapter 42]. We only provide a seemingly clumsy proof of the construction of the second branch $C_{2}^{c}$ so that we could illustrate the pasting technique. On $C_{2}^{c}$ the solution could be split in the positive part $x_{P}(t)$ and negative part $x_{N}(t)$. Without loss of generality, we assume that $x_{P}(t)=\sin \left(\frac{\pi t}{m}\right)$, where $m \in(0, \pi)$. Then the negative part must have the form $x_{N}(t)=C \sin \beta(t-\pi)$, where $\beta=\frac{-\pi}{m-\pi}$ is chosen so that $x_{P}$ and $x_{N}$ meet at $m$. The constant $C$ is then chosen so that this connection is continuously differentiable (cf. Figure 1). From this we have that $\mu=\left(\frac{\pi}{m}\right)^{2}$ and $v=\left(\frac{\pi}{\pi-m}\right)^{2}$, 


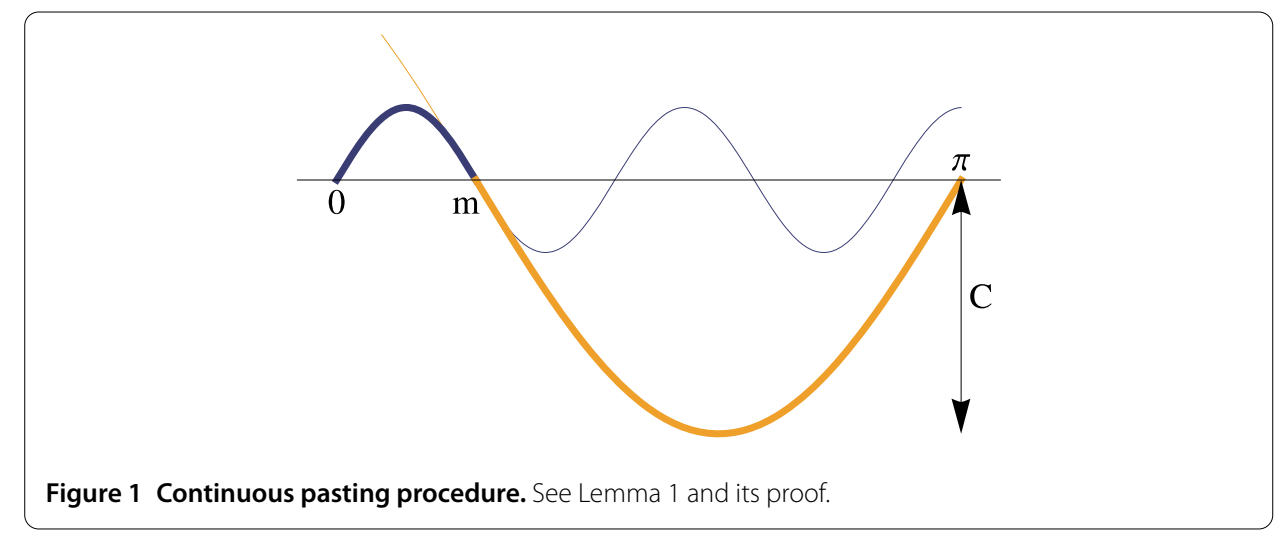

which implies that

$$
\frac{1}{\sqrt{\mu}}+\frac{1}{\sqrt{v}}=\frac{1}{2} \text {. }
$$

\section{Trivial first branch}

Let us return back to the discrete problem (1). Following the continuous notation, we denote the discrete spectrum by $\Sigma^{d}$. We realize first that the problem (1) on $N+2$ points can change sign only $(N-1)$-times, which implies that the spectrum on $N+2$ points will have only $N$ branches $C_{i}^{d}$, i.e.,

$$
\Sigma^{d}=\bigcup_{i=1}^{N} C_{i}^{d} .
$$

Naturally, the first Fučík branch $C_{1}^{d}$ of (1) is given by the eigenvalues of the discrete problem

$$
\left\{\begin{array}{l}
-\Delta^{2} x(k-1)=\lambda x(k), \quad k=1,2, \ldots, N \\
x(0)=0=x(N+1)
\end{array}\right.
$$

As we use the eigenvalues in the sequel, we present a concise proof.

Theorem 2 The eigenvalues of (6) are

$$
\lambda_{i}=4 \sin ^{2} \frac{i \pi}{2(N+1)}, \quad i=1,2, \ldots, N,
$$

and the corresponding eigenvectors are

$$
u_{i}(k)=\sin \frac{k i \pi}{N+1}, \quad i, k=1,2, \ldots, N .
$$

Proof For a fixed $i=1,2, \ldots, N$, direct substitution of (8) into (6) yields

$$
\begin{aligned}
& -\Delta^{2} u_{i}(k-1)=\lambda_{i} u_{i}(k), \\
& -\sin \frac{i(k-1) \pi}{N+1}+2 \sin \frac{i k \pi}{N+1}-\sin \frac{i(k+1) \pi}{N+1}=\lambda_{i} \sin \frac{i k \pi}{N+1},
\end{aligned}
$$




$$
\begin{aligned}
& 2 \sin \frac{i k \pi}{N+1}-2 \sin \frac{i k \pi}{N+1} \cos \frac{i \pi}{N+1}=\lambda_{i} \sin \frac{i k \pi}{N+1}, \\
& 2-\cos \frac{i \pi}{N+1}=\lambda_{i} \\
& 4 \sin ^{2} \frac{i \pi}{2(N+2)}=\lambda_{i} .
\end{aligned}
$$

Thus, we have $N$ independent eigenvectors, which finishes the proof.

The first eigenvalue $\lambda_{1}=4 \sin ^{2} \frac{\pi}{2(N+2)}$ generates the first Fučík branch $C_{1}^{d}$ of (1). Since the corresponding eigenfunction $u_{1}(k)=\sin \frac{k \pi}{N+1}$ does not change its sign, we obtain that the problem (1) has a nontrivial solution $u_{1}(k)$ for an arbitrary couple $\left(\lambda_{1}, v\right)$, with $v \in \mathbb{R}$. Similarly, the problem (1) has a nontrivial solution $-u_{1}(k)$ for an arbitrary couple $\left(\mu, \lambda_{1}\right)$, with $\mu \in \mathbb{R}$.

\section{Second branch and anchoring}

Let us move on to the second discrete Fučík branch $C_{2}^{d}$. It corresponds to the solutions of (1) that change the sign exactly once (see Figure 2 for illustration and basic notation). As in the continuous case, we try to paste together two sine functions. Without loss of generality, we assume that the solution is positive first. We choose $m \in(1, N)$ and consider solutions which are nonnegative on $[0, m]_{\mathbb{Z}},{ }^{\text {a }}$ with values lying on the sine function $(c f$. Theorem 2 and Figure 2)

$$
x_{P}(k)=\sin \left(\frac{\pi}{m} k\right) .
$$

Since the solutions on the second branch change sign exactly once and lie on the sine function again, we seek constants $C, \beta \in \mathbb{R}$ ( $c f$. Theorem 2 and Figure 2$)$ in the nonpositive part

$$
x_{N}(k)=C \sin \beta(k-N-1) .
$$

Then the vector $(\lfloor m\rfloor$ and $\lceil m\rceil$ denote floor and ceiling functions)

$$
x=\left[x_{P}, x_{N}\right]:=\left[0, x_{P}(1), \ldots, x_{P}(\lfloor m\rfloor), x_{N}(\lceil m\rceil), \ldots, x_{N}(N), 0\right]^{T}
$$

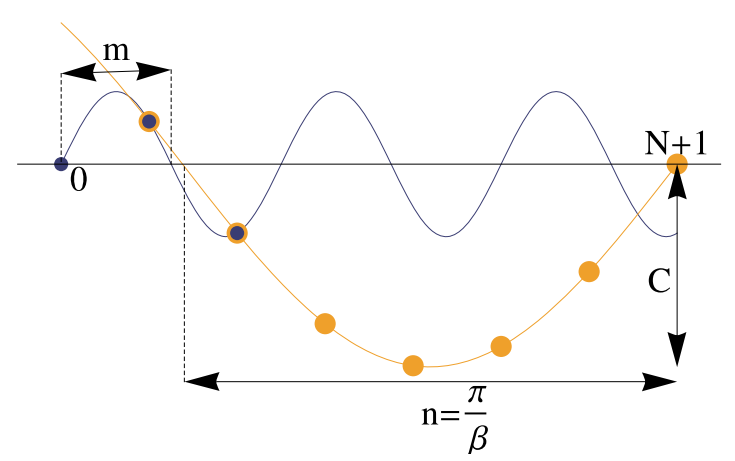

Figure 2 Discrete anchoring procedure. Illustration of the fact that $m+n \neq N+1$ in general. 
is the solution of (1) coupled with the pair $(\mu, v)$ given by

$$
\mu=4 \sin ^{2} \frac{\pi}{2 m}, \quad v=4 \sin ^{2} \frac{\beta}{2} .
$$

Above, we considered $m \in(1, N)$. This follows from the fact that if we had chosen $m \leq 1$ or $m \geq N$, there would have been no positive/negative part and the solution would have laid on $C_{1}^{d}$ instead.

Our first observation is trivial and considers integer values of $m$. In this case, the transition between the positive and negative parts occurs exactly at $m=\lfloor m\rfloor=\lceil m\rceil$, and we could easily compute $\beta$ and $C$ in (10). In other words, we could still talk about pasting in this case.

Lemma 3 If $m \in(1, N)$ is an integer number, then

$$
\beta=\frac{\pi}{N+1-m}, \quad C=\frac{\sin \frac{\pi}{m}}{\sin \frac{\pi}{N+1-m}} .
$$

Moreover, $x_{P}$ and $x_{N}$ are equal in $m-1, m$ and $m+1$.

Proof If $m$ is integer, then $x_{P}(m)=0$. Consequently, the difference equation (1) at $k=m$ reduces to

$$
x_{P}(m-1)+x_{N}(m+1)=0 .
$$

Exploiting the symmetry of sine functions, both $x_{P}$ and $x_{N}$ are equal in $m-1, m$ and $m+1$. Since $x_{N}(m)=0$, we obtain that

$$
\beta=\frac{\pi}{N+1-m} .
$$

Finally, the equality of functions $x_{P}$ and $x_{N}, e . g$., at $m+1$, implies that

$$
\begin{aligned}
& \sin \frac{\pi(m+1)}{m}=C \sin \frac{\pi}{N+1-m}(m+1-N-1), \\
& -\sin \frac{\pi}{m}=-C \sin \frac{\pi}{N+1-m}, \\
& C=\frac{\sin \frac{\pi}{m}}{\sin \frac{\pi}{N+1-m}} .
\end{aligned}
$$

The analysis gets more complicated once we consider non-integer values of $m$. In this case, the transition between positive and negative parts occurs between $\lfloor m\rfloor$ and $\lceil m\rceil$. Our first result states that the values of $x_{P}$ and $x_{N}$ coincide at $\lfloor m\rfloor$ and $\lceil m\rceil$ (cf. Figure 2).

Lemma 4 (Necessary condition) Let $m \in(1, N)$ be non-integer. Let $x=\left[x_{P}, x_{N}\right]$ be a nontrivial solution of (1) with $x_{P}$ and $x_{N}$ given by (9) and (10). Then the following equalities hold:

$$
\begin{aligned}
& x_{P}(\lfloor m\rfloor)=x_{N}(\lfloor m\rfloor), \\
& x_{P}(\lceil m\rceil)=x_{N}(\lceil m\rceil) .
\end{aligned}
$$


Proof If $x_{P}$ is given by (9), then the equation

$$
\Delta^{2} x_{P}(k-1)=\mu x_{P}(k)
$$

holds for $k=1,2, \ldots,\lfloor m\rfloor-1$ if and only if (see the proof of Theorem 2)

$$
\mu=4 \sin ^{2} \frac{\pi}{2 m}
$$

If we consider the difference equation (1) in $\lfloor m\rfloor$ we get

$$
x_{P}(\lfloor m\rfloor-1)-2 x_{P}(\lfloor m\rfloor)+x_{N}(\lceil m\rceil)=4 \sin ^{2} \frac{\pi}{2 m} x_{P}(\lfloor m\rfloor) .
$$

This equality holds if and only if (see (1))

$$
x_{N}(\lceil m\rceil)=x_{P}(\lceil m\rceil),
$$

which verifies (14).

Using the same argument at $\lceil m\rceil$ for $x_{N}$, we obtain that (13) holds as well.

This result enables us to get both peripheral parts of $C_{2}^{d}$.

\section{Corollary 5}

$$
(\mu, v)=\left(4 \sin ^{2} \frac{\pi}{2 m}, 2-\frac{\sin \frac{(N-1) \pi}{m}}{\sin \frac{N \pi}{m}}\right) \in C_{2}^{d}, \quad m \in(N-1, N)
$$

and

$$
(\mu, \nu)=\left(2-\frac{\sin \frac{(N-1) \pi}{n}}{\sin \frac{N \pi}{n}}, 4 \sin ^{2} \frac{\pi}{2 n}\right) \in C_{2}^{d}, \quad n \in(N-1, N) .
$$

Proof Let us consider $m \in(N-1, N)$. Then we have

$$
x_{P}(N-1)=\sin \left(\frac{(N-1) \pi}{m}\right), \quad x_{P}(N)=x_{N}(N)=\sin \left(\frac{N \pi}{m}\right) \text {. }
$$

Then we can rewrite the equation (1) in $k=N$,

$$
\begin{aligned}
& -x(N-1)+2 x(N)-x(N+1)=v x(N), \\
& -\sin \left(\frac{(N-1) \pi}{m}\right)+2 \sin \left(\frac{N \pi}{m}\right)=v \sin \left(\frac{N \pi}{m}\right), \\
& 2-\frac{\sin \frac{(N-1) \pi}{m}}{\sin \frac{N \pi}{m}}=v .
\end{aligned}
$$

This proves the former part of the statement. The latter follows from the mirror argument. 
Obviously, $v>4$ for $m$ sufficiently close to $N$. This implies that (11) cannot hold for any $\beta$, i.e., not all the solutions on the second branch can be obtained as a composition of sine functions!

Since the problem is solved for $m \in(1,2) \cup(N-1, N)$, we could turn our attention to $m \in(2, N-1)$ in the following. Applying (9) and (10), we can rewrite conditions (13) and (14) in the following way.

Corollary 6 (Necessary condition II) Let $m \in(2, N-1)$ be non-integer. If $x_{P}$ and $x_{N}$ have the form (9) and (10), then the following equality is satisfied:

$$
\sin \left(\frac{\pi}{m}\lfloor m\rfloor\right) \sin \beta(\lceil m\rceil-N-1)=\sin \left(\frac{\pi}{m}\lceil m\rceil\right) \sin \beta(\lfloor m\rfloor-N-1) .
$$

Proof One can rewrite equalities (13) and (14) into

$$
\begin{aligned}
& \sin \left(\frac{\pi}{m}\lfloor m\rfloor\right)=C \sin \beta(\lfloor m\rfloor-N-1), \\
& \sin \left(\frac{\pi}{m}\lceil m\rceil\right)=C \sin \beta(\lceil m\rceil-N-1) .
\end{aligned}
$$

Isolating $C$ on the right-hand sides of both equations, we get

$$
\frac{\sin \left(\frac{\pi}{m}\lfloor m\rfloor\right)}{\sin \beta(\lfloor m\rfloor-N-1)}=C=\frac{\sin \left(\frac{\pi}{m}\lceil m\rceil\right)}{\sin \beta(\lceil m\rceil-N-1)} .
$$

Now, it suffices to multiply this equality by both denominators to get (15).

Remark 7 (Anchoring) Corollary 6 implies that the (continuous extensions of) sine functions (9) and (10) do not intersect at $m$ in general. Indeed, we could see that $\beta=\frac{\pi}{N+1-m}$ does not solve (15) for all $m$. In other words, if we define $n:=\frac{\pi}{\beta}$, we have $m+n \neq N+1$ for almost every $m \in(1, N)$ (see Figure 2).

We have shown that

$$
C_{2}^{d}={ }^{\mathbb{Z}} C_{2}^{d} \cup{ }^{1} C_{2}^{d} \cup{ }^{2} C_{2}^{d}
$$

where (see Lemma 3 and (11))

$$
{ }^{\mathbb{Z}} C_{2}^{d}=\left\{(\mu, v): \mu=4 \sin ^{2} \frac{\pi}{2 m}, v=4 \sin ^{2} \frac{\pi}{2(N+1-m)}, m \in(1, N)_{\mathbb{Z}}\right\}
$$

and the parametrization of ${ }^{1} C_{2}^{d}$ is given by (5). Finally, we would like to get the parametrization of ${ }^{2} C_{2}^{d}$, i.e., consider $m \in(2, N-1)$. Corollary 6 implies that one could get the parametrization of ${ }^{2} C_{2}^{d}$ if and only if one could solve (15). Equation (15) is equivalent to the problem of solving the transcendent equation

$$
A \sin (x)=\sin (B x)
$$


with $A<0$ and $B \in \mathbb{Q}$. Indeed, if we define

$$
A=\frac{\sin \left(\frac{\pi}{m}\lfloor m\rfloor\right)}{\sin \left(\frac{\pi}{m}\lceil m\rceil\right)}, \quad C=\lceil m\rceil-N-1, \quad D=\lfloor m\rfloor-N-1,
$$

then the nonlinear equation (15) can be rewritten as

$$
A \sin (C \beta)=\sin (D \beta)
$$

Considering $m \in(\lfloor m\rfloor,\lceil m\rceil)$, we see that $D$ and $C$ are constant non-zero integers and $A \in$ $(-\infty, 0)$. Consequently, if we substitute $x=B \beta$ and $D=B C$, we get (16).

In order to get the parametrization of the complete second Fućík branch $C_{2}^{d}$, we need to solve (15) for $\beta$, or, equivalently, (16) for $x$. While this could be done pretty easily numerically, we state, in the final section, a conjecture that it is not possible to use a finite number of elementary functions to get such a parametrization. Meanwhile, we make two straightforward observations.

Remark 8 Considering non-integer values of $m$, we can solve equation (15) only in the symmetric case, in which $m=\lfloor m\rfloor+\frac{1}{2}=\lceil m\rceil-\frac{1}{2}$. Either the symmetry of sine functions or the direct computation yields that $\beta=\frac{\pi}{N+1-m}$. Similarly, as in the integer case (cf. Lemma 3$)$, this value coincides with the value of the corresponding continuous problem as the positive and negative parts meet at $m$.

Combining this with the following straightforward corollary, we observe that the second branch $C_{2}^{d}$ of the discrete spectrum slides monotonically along its continuous counterpart, coinciding in the 'symmetric' values of $m$, in which $2 m \in \mathbb{N}$ (see Figure 3).

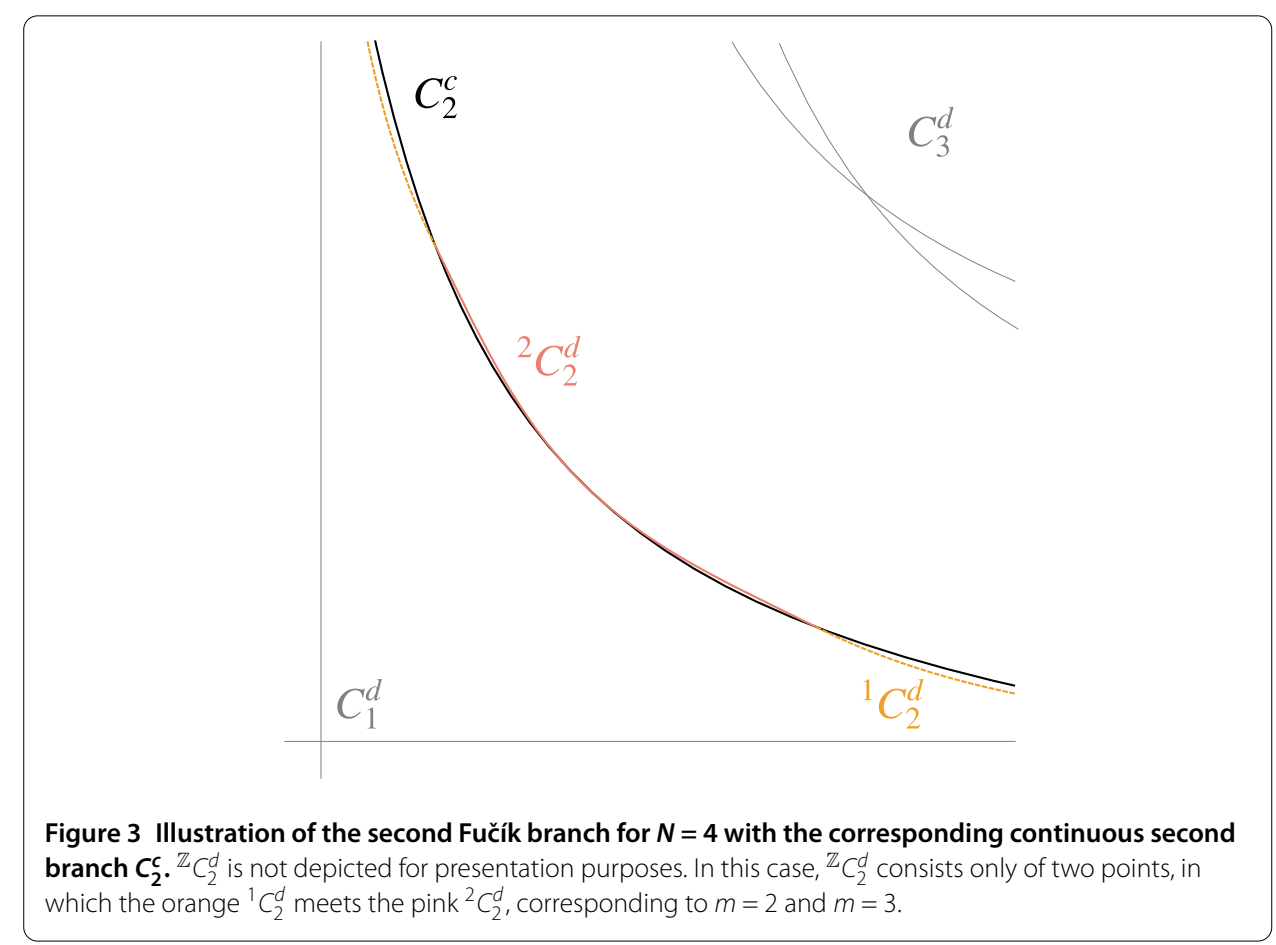


Corollary 9 The value of $v$ is decreasing in $\mu$.

Proof Putting $\beta=\beta(m)$ and differentiating (15), we get

$$
\begin{aligned}
& \beta^{\prime}(m) \\
& =\frac{\cos \left(\frac{\pi}{m}\lfloor m\rfloor\right) \cdot \frac{\pi\lfloor m\rfloor}{m^{2}} \cdot \sin \beta(\lceil m\rceil-N-1)-\cos \left(\frac{\pi}{m}\lceil m\rceil\right) \cdot \frac{\pi\lceil m\rceil}{m^{2}} \cdot \sin \beta(\lfloor m\rfloor-N-1)}{\sin \left(\frac{\pi}{m}\lfloor m\rfloor\right) \cos \beta(\lceil m\rceil-N-1)-\sin \left(\frac{\pi}{m}\lceil m\rceil\right) \cos \beta(\lfloor m\rfloor-N-1)} .
\end{aligned}
$$

Analyzing the signs of individual terms and observing that $\frac{\pi}{m}\lfloor m\rfloor<\pi$ and that $\frac{\pi}{m}\lceil m\rceil>\pi$, we conclude that

$$
\beta^{\prime}(m)>0,
$$

and (11) yields the result.

\section{Elementariness of the second branch $C_{2}^{d}$}

Since the second-branch $C_{2}^{d}$ of (1) is the first nontrivial branch of the simplest discrete Fučík spectrum, we believe that its properties can help to explain difficulties with discrete Fučík spectra. Therefore, we discuss possible ways to prove that its part ${ }^{2} C_{2}^{d}$ has no elementary parametrization.

Definition 10 We say that a function is elementary if it is a finite composition of rational, algebraic, exponential, logarithmic, trigonometric, inverse trigonometric, hyperbolic and inverse hyperbolic functions. We say that a parametrization of a curve in $\mathbb{R}^{2}$ is elementary if it consists of elementary functions.

Under this definition, our conjecture becomes as follows.

Conjecture 11 The second branch $C_{2}^{d}$ of (1) has no elementary parametrization.

Our analysis in the previous section implies that one could rephrase this conjecture in the following way.

Conjecture 12 The solution of equation (16) cannot be solved in elementary functions.

Since there is a developed theory of elementary integration (see [14]) and (to our knowledge) there is no suitable tool dealing with elementary parametrizations of transcendent equations like (16), we try to use the theory of elementary integration to attack Conjectures 11 and 12.

Definition 13 We say that the integral is elementary if it can be expressed in terms of elementary functions.

One could use the Risch algorithm [14, Chapter 12] to determine whether an integral is elementary or not. We use this procedure to analyze an integral directly connected to (15).

Lemma 14 The integral $\int \frac{\mathrm{d} x}{x \sin (x)}$ is not elementary. 
Proof Denoting $\theta=e^{i x}, a(\theta)=2 i \theta$ and $b(\theta)=x\left(\theta^{2}-1\right)$, we rewrite the integral

$$
\int \frac{\mathrm{d} x}{x \sin (x)}=\int \frac{2 i \theta}{x\left(\theta^{2}-1\right)} \mathrm{d} x=\int \frac{a(\theta)}{b(\theta)} \mathrm{d} x .
$$

We compute the following resultant (see [14, Definition 7.3]):

$$
\begin{aligned}
R(z) & =\operatorname{res}_{\theta}\left(a(\theta)-z \cdot b^{\prime}(\theta), b(\theta)\right) \\
& =\operatorname{res}_{\theta}\left(2 i \theta-z \theta^{2}(1+2 i x)+z, x\left(\theta^{2}-1\right)\right) \\
& =\left|\begin{array}{cccc}
-z(1+2 i x) & 2 i & z & 0 \\
0 & -z(1+2 i x) & 2 i & z \\
x & 0 & -x & 0 \\
0 & x & 0 & -x
\end{array}\right| \\
& =4 x^{2}-4 x^{4} z^{2} .
\end{aligned}
$$

Since the roots of $R(z)$ are $z= \pm \frac{1}{x}$, i.e., not constant, the integral is not elementary (see the Rothstein-Trager theorem [14, Theorem 12.9]).

Let us return back to Conjecture 12 and study (16) in more general settings, considering $A, B \in \mathbb{R}$.

Conjecture 15 Equation $x \sin (u)-\sin (y u)=0$ cannot be solved for $u=u(x, y)$ using a finite number of elementary functions.

Let us denote $f(x, y, u):=x \sin (u)-\sin (y u)$ and consider the equation $f(x, y, u)=0$. Since $\frac{\partial f}{\partial u}=x \cos (u)-y \cos (y u)$, the implicit function theorem can be applied in points $(x, y, u)$ such that $x \sin (u)=\sin (y u)$ and $x \cos (u) \neq y \cos (y u)$. Therefore, we take into account triplets $(x, y, z)=\left(1, C, \frac{2 \pi n}{1-C}\right)$ with $C \neq 1, n \in \mathbb{Z}$.

The implicit function theorem implies that the solution $u=u(x, y)$ starting in those points satisfies

$$
\begin{aligned}
& 0=\frac{\partial}{\partial x} f(x, y, u(x, y))=\sin (u)+[x \cos (u)-y \cos (y u)] \frac{\partial u}{\partial x}, \\
& 0=\frac{\partial}{\partial y} f(x, y, u(x, y))=-u \cos (y u)+[x \cos (u)-y \cos (y u)] \frac{\partial u}{\partial y} .
\end{aligned}
$$

Multiply the first equation by $x u \cos (u)$, the second equation by $y \sin (u)$, add both and divide the result by the non-zero term in the square brackets to obtain the initial problem for the first-order partial differential equation $(y \neq 1)$

$$
\left\{\begin{array}{l}
x u \cos (u) \frac{\partial u}{\partial x}+y \sin (u) \frac{\partial u}{\partial y}=-u \sin (u), \\
u(1, y)=\frac{2 \pi n}{1-y}
\end{array}\right.
$$

Characteristic equations of (17) are given by

$$
\left\{\begin{array}{l}
\dot{x}=x z \cos (z), \quad x(0)=1, \\
\dot{y}=y \sin (z), \quad y(0)=C, C \neq 1 \\
\dot{z}=-z \sin (z), \quad z(0)=\frac{2 \pi n}{1-C}, n \in \mathbb{Z}
\end{array}\right.
$$


where $z=u(x, y)$ is the value of $u$ along characteristic curves. The third equation of (18) cannot be solved using elementary functions for $n \neq 0$ as the integral $\int \frac{1}{z \sin (z)} \mathrm{d} z$ is not elementary (Lemma 14).

Unfortunately, the existence of non-elementary parametrization does not imply yet that the solution surfaces $u=u_{n}(x, y)$ of $f(x, y, u)=0$ (which arise as a union of the characteristic curves) cannot be expressed using elementary functions.

\section{Conclusion}

The goal of this paper was to shed some light on the problems which have arisen in the study of the discrete Fučík spectrum or related resonance problems. Although we were unable to fully prove the nonexistence of elementary parametrization of the second branch of the simplest discrete Fučík spectrum, we believe that the anchoring technique and the relationship to the transcendent equation (16) help to understand better the troubles which occur in this area.

\section{Competing interests}

The author declares that they have no competing interests.

\section{Acknowledgements}

This research brought the author to the corners of mathematics he had not been familiar with. Therefore, he is very thankful to Jochen Merker and Petr Nečesal for their guidance. His thanks are also directed to Pavel Drábek, Gabriela Holubová and Komil Kuliev. This research has been supported by the grants of Ministry of Education, Youth and Sports of the Czech Republic ME09109 and MSM 4977751301.

\section{Endnote}

a Subscript $\mathbb{Z}$ denotes the discrete interval, i.e., $[0, m]_{\mathbb{Z}}:=[0, m] \cap \mathbb{Z}$.

Received: 23 November 2012 Accepted: 8 March 2013 Published: 29 March 2013

References

1. Bereanu, C, Mawhin, J: Existence and multiplicity results for periodic solutions of nonlinear difference equations. J. Differ. Equ. Appl. 12, 677-695 (2006)

2. Galewski, M: Dependence on parameters for discrete second-order boundary value problems. J. Differ. Equ. Appl. 17 1441-1453 (2011)

3. Stehlík, P: On variational methods for periodic discrete problems. J. Differ. Equ. Appl. 14(3), 259-273 (2008)

4. Fučík, S: Boundary value problems with jumping nonlinearities. Čas. Pěst. Mat. 101, 69-87 (1976)

5. Dancer, EN: On the Dirichlet problem for weakly nonlinear elliptic partial differential equations. Proc. R. Soc. Edinb. 293, 187-197 (1999)

6. Fučík, S: Solvability of Nonlinear Equations and Boundary Value Problems. Reidel, Dordrecht (1980)

7. Drábek, P, Robinson, S: Resonance problems for the $p$-Laplacian. J. Funct. Anal. 169(1), 189-200 (1999)

8. Rynne, B: The Fucik spectrum of general Sturm-Liouville problems. J. Differ. Equ. 161(1), 87-109 (2000)

9. Ben Naoum, A, Fabry, C, Smets, D: Structure of the Fučík spectrum and existence of solutions for equations with asymmetric nonlinearities. Proc. R. Soc. Edinb. 131(2), 241-265 (2001)

10. Holubová, G, Nečesal, P: The Fučík spectrum: exploring the bridge between discrete and continuous world (in press)

11. Ma, R, Xu, Y, Gao, C: Spectrum of linear difference operators and the solvability of nonlinear discrete problems. Discrete Dyn. Nat. Soc. 2010, Article ID 757416 (2010)

12. Margulies, C, Margulies, W: Nonlinear resonance set for nonlinear matrix equations. Linear Algebra Appl. 293, 187-197 (1999)

13. Švarc, R: Two examples of the operators with jumping nonlinearities. Comment. Math. Univ. Carol. 30(3), 587-620 (1989)

14. Geddes, KO, Czapor, SR, Labahn, G: Algorithms for Computer Algebra. Kluwer, Dordrecht (1992) 